\title{
Flexible Ethikgremien. Impulse zur Institutionalisierung ethisch verantwortlicher Feldforschung in der Konflikt- und Fluchtforschung
}

\author{
Ulrike Krause • Timothy Williams
}

Online publiziert: 14. August 2020

(C) Der/die Autor(en) 2020

Zusammenfassung Forschungsvorhaben in der Konflikt- und Fluchtforschung finden häufig in schwierigen Kontexten statt und erfordern forschungsethische Reflexionen. Welche Bedeutungen kommen Ethikkommissionen und -prüfungen zur Förderung und Sicherstellung von ethisch verantwortlicher Forschung in diesen Bereichen zu? Mit dieser Ausgangsfrage setzen wir uns in dem Beitrag auseinander und kontrastieren Argumente für und gegen standardisierte Kommissionen und Prüfungen. Da sich Kritiken häufig auf zu starke Kontrollen solcher Kommissionen richten, Forschende aber den Austausch als relevant erachten, schlagen wir die Einrichtung und Nutzung von flexiblen interdisziplinären Ethikgremien vor. Diese Gremien würden nicht die Prüfungen, sondern vielmehr den Austausch über forschungsethische Fragen in den Mittelpunkt rücken. Mithilfe empirischer Beispiele zum Thema Sicherheit, konkret zur Forschung über genderbasierte Gewalt und zum Umgang mit sexueller Orientierung von Forschenden und Teilnehmenden reflektieren wir die Notwendigkeit ethisch verantwortlicher Forschung sowie den möglichen Mehrwert flexibler Ethikgremien.

\section{Flexible Ethics Boards. Some Thoughts on Institutionalizing Ethically Responsible Field Research in Conflict and Forced Migration Studies}

\footnotetext{
Abstract Research projects in conflict and forced migration studies usually take place in difficult contexts and thus require reflections on research ethics. What

U. Krause $(\bowtie)$

Institut für Migrationsforschung und Interkulturelle Studien (IMIS), Institut für Sozialwissenschaften, Universität Osnabrück, Neuer Graben 19/21, 49069 Osnabrück, Deutschland

E-Mail: ulrike.krause@uni-osnabrueck.de

T. Williams

Fakultät für Staats- und Sozialwissenschaften, Institut für Politikwissenschaft, Universität der Bundeswehr München, Werner-Heisenberg-Weg 39, 85577 Neubiberg, Deutschland
} 
importance do ethics committees and reviews have in promoting or ensuring ethically responsible research in these areas? This article deals with this question by discussing arguments for and against standardized ethics committees and review processes, such as institutional review boards and institutionalised processes. As most critiques focus on the too controlling nature of these committees, but most researchers see exchange as fruitful, we suggest the creation and implementation of flexible interdisciplinary ethics boards that do not primarily restrict, but focus on providing space for discussion about research ethical questions. By reflecting on empirical examples of security, in particular concerning gender-based violence and sexual orientation of researchers and participants, we reflect on the need for ethically responsible research as well as the potential value of flexible ethics boards.

\section{Einleitung}

Wir verzeichnen jüngst einen „Feldforschungs-Boom“ (Menzel 2014, S. 280) in den sozialwissenschaftlich ausgerichteten Feldern der Friedens- und Konfliktforschung sowie der Flucht- und Flüchtlingsforschung (im Folgenden kurz: Konfliktund Fluchtforschung), welcher eine kritische Reflexion der Notwendigkeit wie auch der Art und Weise ihrer Umsetzung erfordert. Während die Notwendigkeit von ,Feldbesuchen ${ }^{1}$ grundsätzlich von den jeweiligen Forschungslücken und -fragen abhängt, ist die Art und Weise, wie ,im Feld“ geforscht wird, nicht auf Fragen von Methoden und Feldzugang beschränkt. Gerade in Bereichen wie der Konflikt- und Fluchtforschung sind forschungsethische Abwägungen notwendig, da die Studien oft in herausfordernden Umfeldern stattfinden und Menschen involvieren, die Gewalt direkt oder indirekt erfahren oder ausgeübt haben. Es ist unabdingbar zu ermessen, welche Implikationen Forschungsaktivitäten haben und wie ethische Standards eingehalten werden können.

Da Forschungsvorhaben in höchst unterschiedlichen Kontexten und mit diversen Personen stattfinden, gibt es keine Universallösung für die Einhaltung ethischer Standards. Vielmehr verstehen wir Forschungsethik vordergründig als normengeleitete Reflexion der Zugänge und Methoden zur Datenerhebung und -auswertung. Im Zentrum gilt es, Nutzen und Wirkungen verschiedener Ansätze abzuwägen und zu eruieren, wie Forschende Beziehungen mit Teilnehmenden aufbauen und Vertrauen bilden und vor allem wie sie potenzielle negative Konsequenzen der Forschung (z. B. Gefahren oder Unannehmlichkeiten) für Teilnehmende und Forschende vermeiden können. Anhand dieser Abwägung wird eine ethisch verantwortliche Durchführung empirischer Feldforschung befördert.

\footnotetext{
1 Gemeinhin verstehen wir ,Feldforschung ' als das Sammeln von Primärdaten in spezifischen Kontexten außerhalb von Labors. Dadurch werden sowohl Lebensrealitäten, Erfahrungen, Einstellungen und Praxen von Menschen und Gruppen, Organisationen und Institutionen als auch die kulturellen, sozialen, politischen, ökonomischen und ökologischen Umwelten, in denen diese eingebettet sind, erhoben und untersucht. Wie diese Forschung ausgestaltet ist, hängt maßgeblich vom disziplinären Hintergrund der Forschenden ab und kann viele unterschiedliche Methoden wie Interviews, teilnehmende Beobachtung, Umfragen oder Experimente umfassen.
} 
Diese Aspekte sind keinesfalls neu. Zur Wahrung ethischer Standards wurden einerseits forschungsethische Richtlinien für diverse Forschungsdisziplinen und -felder von entsprechenden Dachverbänden oder Instituten entwickelt. ${ }^{2}$ Diese Richtlinien stellen Forschungsethik primär als fundamentale Verhaltenskodizes für Forschende in der wissenschaftlichen Auseinandersetzung und im Umgang mit Teilnehmenden und der Umgebung dar. Zumeist zielen sie auf die Minimierung möglicher Schädigungen für die Untersuchungssubjekte ab und Forschende setzen sie nach eigenem Ermessen um. Während wir in der internationalen Konfliktforschung forschungsethische Angaben vermissen, ${ }^{3}$ lässt sich für die Fluchtforschung etwa auf den ,code of ethics ${ }^{6}$ der International Association for the Study of Forced Migration (IASFM 2018), die Richtlinien kanadischer Verbände (Clark-Kazak et al. 2017) oder auch institutsinterne Leitlinien (u. a. Refugee Studies Centre 2007) verweisen. Doch diese Beispiele stammen aus internationalen bzw. englischsprachigen Umgebungen. Obwohl einige sozialwissenschaftliche Vereinigungen ${ }^{4}$ und universitäre Fachbereiche und Institute ${ }^{5}$ in Deutschland Ethikrichtlinien eingeführt haben, fehlen sie anderenorts häufig. Dies trifft bedauerlicherweise auch auf die Felder der Konflikt- und der Fluchtforschung in Deutschland zu - weder zentrale Institute noch die entsprechenden Dachverbände der Arbeitsgemeinschaft für Friedensund Konfliktforschung e.V. und des Netzwerks Fluchtforschung e.V. haben bisher forschungsethische Richtlinien formuliert. ${ }^{6}$

Andererseits haben sich insbesondere im angelsächsischen Raum Ethikkommissionen oder andere Arten von ,Institutional Review Boards' durchgesetzt (für Entwicklungen zu Ethikkomissionen, vgl. von Unger und Simon 2016). Sie dienen gemeinhin der standardisierten Überprüfung und Kontrolle empirischer Forschungsvorhaben, doch - wie wir in unserem Beitrag zeigen - reflektieren Sozialwissen-

\footnotetext{
2 So haben etwa die American Political Science Association (APSA 2012), die British Sociological Association (BSA 2017) und die American Anthropological Association (AAA 2012), die hier beispielhaft für verschiedene Disziplinen genannt sind, entsprechende Richtlinien.

3 Weder in der International Association for Peace and Conflict Studies noch in der Peace and Justice Studies Association lassen sich entsprechende Angaben finden.
}

${ }^{4}$ Z.B. Deutsche Gesellschaft für Sozial- und Kulturanthropologie (Arbeitsgruppe zu Ethik) (DGSKA 2019), Deutsche Gesellschaft für Soziologie (Ethik-Kodex und Ethikkommission) (DGS 2017) und Deutsche Gesellschaft für Erziehungswissenschaften (Ethik-Kodex) (DGfE 2016).

5 Z.B. Fachbereich Erziehungswissenschaften, Philipps-Universität Marburg; Fachbereich Erziehungsund Sozialwissenschaften, Universität Hildesheim; Fakultät für Human- und Sozialwissenschaften, TU Chemnitz; Fachbereich Sozialwissenschaften, TU Darmstadt.

${ }^{6}$ Zur Konfliktforschung haben wir folgende Einrichtungen berücksichtigt: Zentrum für Konfliktforschung, Philipps-Universität Marburg; Arbeitsbereich Internationale Beziehungen/Friedens- und Konfliktforschung, Universität Tübingen; Institut für interdisziplinäre Konflikt- und Gewaltforschung, Universität Bielefeld; Institut für Friedensforschung und Sicherheitspolitik, Universität Hamburg; Fachbereich Politik- und Verwaltungswissenschaft, Universität Konstanz. Das Bonn International Center for Conversion verweist auf der Internetseite auf DFG-Richtlinien für gute Wissenschaftliche Praxis. Da sich die Fluchtforschung in der deutschen Wissenschaftslandschaft derzeit noch in der Institutionalisierung befindet, haben wir diesbezüglich Institute zur Migrationsforschung berücksichtigt, die sich jüngst stark in der Fluchtforschung engagierten: Institut für Migrationsforschung und Interkulturelle Studien der Universität Osnabrück; Interdisziplinäres Zentrum für Integrations- und Migrationsforschung, Universität DuisburgEssen; Berliner Institut für empirische Integrations- und Migrationsforschung, HU Berlin; Zentrum Flucht und Migration der Katholischen Universität Eichstätt-Ingolstadt; DeZIM-Institut (Stand April 2020). 
schaftler*innen diese Kommissionen durchaus kritisch. In Deutschland gestaltet sich auch hier die Lage anders. Bislang haben vornehmlich Forschende in medizinischen und psychologischen Bereichen Ethikkommissionen zu konsultieren, wenn sie mit Proband*innen arbeiten. Sozialwissenschaftlich verortete Forschende können hingegen solche Prüfungen für ihre Projekte durchlaufen, müssen es aber in den meisten Fällen nicht (von Unger et al. 2014, S. 31); manchenorts gibt es aufgrund fehlender Strukturen nicht einmal die Möglichkeit einer fakultativen Überprüfung. Doch ist dieser Kontrollschritt erforderlich für eine ethisch verantwortliche Durchführung empirischer Forschung? Welche Argumente bestehen für oder gegen institutionalisierte Ethikkommissionen?

In diesem Beitrag reflektieren wir Nutzen und Beschränkungen solcher standardisierten Kommissionen aus der Perspektive sozialwissenschaftlicher Konflikt- und Fluchtforschung. Zur Diskussion bedienen wir uns zunächst empirischer Beispiele eigener wie auch weiterführender Erfahrungen mit einer Gefährdung der Sicherheit, konkret im Kontext der Forschung zu genderbasierter Gewalt und im Umgang mit sexueller Orientierung von Forschenden und Teilnehmenden. Auf Grundlage dieser Beispiele betrachten wir das Für und Wider von standardisierten Kommissionen, d.h. institutionalisierte Kommissionen, die standardisierte Werkzeuge zur forschungsethischen Überprüfung von Projekten nutzen. Entgegen eindringlicher Kritik an den Kommissionen insbesondere aus dem angelsächsischen Raum, dass diese einen bürokratischen Aufwand darstellten sowie unflexibel und beschränkend seien, betonen Wissenschaftler*innen auch einen Mehrwert durch forschungsethische Abwägungen. Vor diesem Hintergrund plädieren wir im Folgenden für eine bestimmte Art: flexible interdisziplinäre Ethikgremien. Wiederum anknüpfend an die empirischen Beispiele sehen wir in den Gremien das Potenzial, dass sie eine gründliche Auseinandersetzung mit Forschungsethik anstoßen und unterstützen können, weniger aber in bürokratischen, stark formalisierten und mitunter einschränkenden Schritten verfangen sind.

\section{Relevanz durch Austausch? Beispiele zur Gefährdung der Sicherheit von Teilnehmenden und Forschenden}

Feldforschung ist in sozialwissenschaftlichen Bereichen generell überaus divers. Dies trifft selbstredend auch auf die Felder der Konflikt- und Fluchtforschung zu. Die Forschung kann weltweit stattfinden, auf diverse Fragen konzentriert sein und anhand vielfältiger Methoden durchgeführt werden. Bezüglich der Konflikt- und Fluchtforschung kann sie etwa die Arbeit mit von Konflikten betroffenen Menschen in Postkonfliktregionen oder mit geflüchteten Menschen in Aufnahmelagern bedeuten. Ein inhärent wichtiges Thema in forschungsethischen Diskussionen wie auch Richtlinien ist die Sicherheit von Teilnehmenden und Forschenden, welches wir in diesem Beitrag im Hinblick auf die Wirkungsmacht von Ethikkommissionen in der Vorbeugung von Problemen diskutieren. Moniert wird in forschungsethischen Diskussionen beispielsweise, dass die Interaktion mit ,westlichen ' Forschenden Gefahren für Teilnehmende bergen kann, insbesondere wenn Autoritätspersonen gegen die Forschung Einwände erheben und Menschen für ihre Teilnahme bestrafen würden 
(Jacobsen und Landau 2003, S. 192-194). Bei einer unausgewogenen oder unreflektierten Wahl von Teilnehmenden, etwa nur einer ethnischen Gruppe, oder bei unsensiblem Umgang mit ihnen oder gar unfreiwilliger Teilnahme können konkrete Sicherheitsprobleme wie auch Angst und Frustration bei den Menschen aufkommen (Ellis et al. 2007; Hugman et al. 2011a; Mackenzie et al. 2007). Strikte topdown Machtdynamiken von Forschenden gegenüber ,Erforschten“ drohen, ihnen Entscheidungen aufzuerlegen und sie mit möglicherweise ungenügendem Raum zur Darstellung ihrer Erfahrungen zu objektivieren (Doná 2007; Pittaway et al. 2010). Weiter kritisieren Wissenschaftler*innen, dass mögliche Sicherheitsimplikationen durch Bezahlung von Befragten entstehen können (Mackenzie et al. 2007), oder dass unzureichende Methoden zur Datenerhebung mit Geflüchteten genutzt werden, um der „Ground Truth“ nachzugehen, dabei aber Gefahren für die Menschen hervorgerufen werden können (Jacobsen und Landau 2003, S. 188).

Während es wie zuvor dargelegt keine pauschalen ,allumfassend richtigen“ Vorgehensweisen gibt, welche diese und weitere Gefahren beseitigen, sind Reflexionen über Strategien des Umgangs damit wichtig. Häufig wird in akademischen Debatten auf den forschungsethischen Ansatz ,Do No Harm ‘ als goldene Regel und normatives Leitprinzip verwiesen, um Sicherheit von Forschenden und Teilnehmenden zu gewährleisten (vgl. u. a. Banks et al. 2013; Brounéus 2011; Browne und Moffett 2014; Fluehr-Lobban 2014; Gerver 2013; Goodhand 2000; Marlowe et al. 2015; Siegel und de Wildt 2016; Van Liempt und Bilger 2012; Vervliet et al. 2015; Wood 2006). Jedoch kritisieren Forschende mitunter, dass dies als Prinzip wenig Wirkungskraft entfaltet und bislang selten operationalisiert wird (zu einer Möglichkeit der Operationalisierung von ,Do No Harm“, vgl. Krause 2017). Es bleibt als selten definiertes Wunschbekenntnis bestehen und droht, zum Lippenbekenntnis zu verkommen und entstehende Gefahren für Teilnehmende und Forschende zu überdecken (krit. vgl. Krause 2017). Forschende wie Hugman et al. (2011b) und Mackenzie et al. (2007) sprechen sich nicht zuletzt deswegen dafür aus, über dieses Leitprinzip hinauszugehen.

Um deutlicher aufzuzeigen, inwieweit Fragen der Sicherheit nur unzulänglich von herkömmlichen Ethikkommissionen und üblichen ,Do No Harm‘ Paradigmen reflektiert werden können, möchten wir nun konkret zwei Beispiele etwas ausführlicher diskutieren. Hier fragen wir, welche besonderen Bedingungen und potenzielle physische und psychische Gefahren sich mit Blick auf sexuelle Orientierung von Forschenden und genderbasierte Gefahren für Teilnehmende ergeben. Bieten standardisierte Kommissionen einen Mehrwert, um diese Risiken zu erkennen und ihnen entgegenzuwirken? In den beiden Illustrationen greifen wir auf eigene Forschungserfahrungen zurück und reflektieren darauf aufbauend Bedeutungen und Begrenzungen von Ethikkommissionen. In der Konsequenz schlagen wir flexible Gremien vor.

Die Erforschung von genderbasierter Gewalt in Konflikt- und Fluchtsituationen stellt mittlerweile kein wissenschaftliches Neuland mehr dar - nichtsdestoweniger sind forschungsethische Reflexionen natürlich zentral. ${ }^{7}$ Von 2013 bis 2016 wurde

\footnotetext{
7 Für einen Überblick zu themenbezogenen Artikeln aus forschungsethischer Perspektive, siehe https:// www.svri.org/research-methods/ethics/journal-abstracts?link-section=journal-articles; Zugegriffen: 24. Juni 2020.
} 
das Projekt „Genderbeziehungen im begrenzten Raum“ umgesetzt, das Bedingungen, Ausmaß und Formen von sexueller und genderbasierter Gewalt an Frauen in Flüchtlingslagern mit Fallstudie in Uganda untersucht hat. ${ }^{8}$ Obwohl das Projekt Gewalt an Frauen in den Fokus nahm, bestand auch Interesse am Verständnis von entsprechenden Gefahren für andere Gruppen und insbesondere für Männer. Denn anstatt einer möglichen Reproduktion binärer Muster von weiblichen Opfern und männlichen Tätern sollte die Komplexität des Themas aufgedeckt werden. Die Feldforschung fand jedoch 2014 zu einem Zeitpunkt statt, als in Uganda ein Gesetz gegen Homosexualität (,anti-homosexuality bill' ${ }^{\circ}$ ) erlassen wurde, das Beziehungen zwischen Lesben, Schwulen, Bisexuellen und Transgender (LGBT) kriminalisiert hat. Die Wirkung des Gesetzes war weitreichend. Intensive politische, mediale und gesellschaftliche Debatten trugen zu Spannungen und einer deutlichen Zunahme an öffentlichen, gewaltsamen Übergriffen auf LGBT- und andere Personen bei (vgl. Makofane et al. 2014). Obwohl die Forschung in einem abgelegenen Flüchtlingslager stattfand, waren diese Spannungen auch dort spürbar. So kamen im Zuge der Planung und der Frühphase der Feldforschung starke Befürchtungen auf, dass für männliche und LGBT-Teilnehmende, die über Gefahren oder Erfahrungen der Gewalt berichten, negative Konsequenzen folgen könnten. Denn sexuelle Gewalt an Männern und LGBT-Personen wird nicht selten als homosexuelle Handlung ausgelegt und es wird dem Opfer Schuld zugeschrieben (vgl. Sivakumaran 2005; zu Diskursen siehe Schulz 2018). Aufgrund der schwierigen politischen Lage hätte dies Teilnehmende in gefährliche Positionen bringen können. Nach einigen Diskussionen zwischen den Teammitgliedern des Forschungsprojekts, in denen Nutzen und Risiken abgewogen wurden, haben wir die Sicherheit der Teilnehmenden priorisiert und beschlossen, im ursprünglichen Fokus auf Frauen zu bleiben. Daher haben wir andere Gruppen nicht proaktiv hinsichtlich sexuellen Gewaltgefahren befragt, sind allerdings in Gesprächsverläufen offengeblieben, insofern Teilnehmende von sich aus Erfahrungen teilen wollten. Andere genderspezifische Gefahren wie strukturelle Gewalt haben wir hingegen mit allen Teilnehmenden thematisiert.

Darüber hinaus spielten forschungsethische Erwägungen selbstverständlich generell und so auch in der Arbeit mit Frauen eine zentrale Rolle. Zusätzlich zur Wahl von Methoden (vgl. Glammeier 2016; Hagemann-White 2016; Helfferich 2016) fragten wir uns: Wie können wir Daten erheben, ohne möglicherweise retraumatisierend $\mathrm{zu}$ wirken oder zu stark in Privatsphären einzugreifen? Wie können wir freiwillige und informierte Teilnahme sicherstellen? Diese Fragen haben wir wiederkehrend und forschungsbegleitend besprochen - auch mit Geflüchteten selbst, um Tendenzen zu vermeiden, die Pittaway und Bartolomei (2013) sowie Pittaway et al. (2010) auf den Punkt bringen. Sie zitieren Geflüchtete in asiatischen Ländern, die Forschungsprozesse stark kritisieren, etwa dass Forschende Geflüchtete gebeten haben, sie zu vergewaltigten Frauen zu bringen, um sie zu interviewen im Sinne von „Tell us what happened - how did you feel?“ (Pittaway et al. 2010, S. 236). Da Ulrike Krause vor dem Forschungsprojekt mehrere Jahre in Uganda gelebt und auch mit Geflüchteten zusammengearbeitet hatte, war sie mit den lokalen Bedingungen

\footnotetext{
${ }^{8}$ Das Projekt wurde von Susanne Buckley-Zistel geleitet und der Deutschen Stiftung Friedensforschung gefördert.
} 
vertraut. Zudem war eine der lokalen Mitarbeiterinnen eine klinische Psychologin mit mehrjähriger Erfahrung in der Arbeit mit Gewaltopfern, was für die Forschung wie auch die Reflexionen extrem zuträglich war.

Themen wie Lebensbedingungen in Uganda, humanitäre Strukturen im Lager oder auch möglichen Gewalterfahrungen wurden nicht direkt, sondern stets in ,dritter Person' adressiert und in seltenen Fällen als direkte Fragen formuliert, um Gespräche nicht eng zu rahmen. Vielmehr war uns wichtig, Tendenzen im Gesprächsverlauf zu schaffen, viel Raum zu lassen, damit die Menschen über die für sie wichtigen Themen sprechen können, und natürlich allem voran Vertrauen zu bilden und die Privatsphäre zu achten. Denn einhergehend mit Malkkis (1995, S. 51) Kritik sahen wir es auch als relevant an, ,to leave some stones unturned, to listen to what my informants deemed important“". Auf Gewalt im Allgemeinen sowie sexuelle und genderbasierte Gewalt im Spezifischen gingen die Menschen häufig frühzeitig von sich aus in Gesprächen ein. Dass dies nicht von vorherigem Austausch über das Forschungsprojekt und dessen Ziele zum Erhalt des Einverständnisses geleitet war, lässt sich darauf zurückführen, dass wir Gespräche offen begannen. Wir stellten das Projekt sehr generell (d.h. ohne Gewaltbezug) vor und fragten die Menschen, ob es für sie in Ordnung sei, wenn wir die konkreten Projektziele am Ende vorstellten, um ihre Antworten nicht in eine Richtung zu leiten. Dem stimmten alle Teilnehmenden zu. Dieses Vorgehen war ferner zentral, weil wir auch am Ausmaß der Gewalt interessiert waren. Für das Einverständnis zur Teilnahme nutzten wir einen ähnlich relationalen Prozess, wie er von Mackenzie et al. (2007, S. 306-312) vorgeschlagen wird. Diesem Ansatz liegt der Gedanke zugrunde, dass Einverständnis nicht allein durch eine einmalige Zustimmung (etwa durch das Unterzeichnen einer Einverständniserklärung) erreicht werden könne. Indem Mackenzie et al. an die Handlungsmacht von Teilnehmenden anknüpfen und diese ernst nehmen, schlagen sie vielmehr als angemesseneren Weg vor, Vereinbarungen in fortlaufenden Verhandlungen zu treffen, in denen die Teilnahmebedingungen kontinuierlich besprochen werden und die Teilnehmenden so eine gewisse Kontrolle über die Forschungsverfahren sowie die Art und Weise ihres Engagements behalten.

Zusätzlich zu Sicherheitsdilemmata für Teilnehmende können natürlich auch Wissenschaftler*innen mit Gefahren konfrontiert sein. Diese betreffen nicht nur möglicherweise schwierige Kontextbedingungen und alltägliche Risiken (z.B. Transportmittel, Unterkunft oder Krankheiten), sondern auch Gefahren für gewaltsame Übergriffe. Damit einhergehend betont Darling, dass ,das Feld“ „eine beängstigende, anspruchsvolle und manchmal verwirrende Erfahrung sein kann, wobei Forschende eine Vielzahl von Annahmen, Erwartungen und Motivationen aushandeln müssen“ (übers. von Verf., Darling 2014, S. 201). Dies ist unter Umständen emotional herausfordernd (Thomson et al. 2013), psychisch belastend und physisch gefährlich. Letzteres bringt Luisa Schneider (2020) anhand ihrer Erfahrungen auf den Punkt und reflektiert die daraus entstehenden Herausforderungen in ihrer wissenschaftlichen Arbeit.

Im Kontext unseres Fokus auf sexuelle Orientierung stehen hier die Erfahrungen des zweiten Autors während seiner Feldforschung in Ruanda im Mittelpunkt. Als homosexueller Mann ist seine sexuelle Orientierung in einem Land, in welchem Homosexualität kriminalisiert und sozial geächtet ist, eine deutliche Gefahrenquelle. 
Während ein Verzicht auf homosexuelle Handlungen während der Feldforschungszeit juristische Probleme ausschließen kann, sind soziale Konsequenzen aufgrund seiner Identität nicht ausgeschlossen. Obwohl gewaltsame Übergriffe auf Homosexuelle in Ruanda weniger ausgeprägt sind als im benachbarten Uganda, finden sie nichtsdestotrotz statt. Für einen weißen Forscher aus einem Land im globalen Norden ist es zudem unwahrscheinlicher, dass er Ziel von Gewalthandlungen wird was selbstverständlich zahlreiche weiterführende Machtfragen hervorruft, auf die wir hier nicht eingehen können. Dennoch ist eine Sicherheitsgefährdung in einem Kontext solch starker Diskriminierung und so ausgeprägter Homophobie nicht gänzlich ausgeschlossen. Der Forscher hat daher keiner ruandischen Person während seines Aufenthalts irgendwelche Indizien über seine sexuelle Orientierung gegeben, von (ehemaligen) Beziehungspartnern wurde verweiblicht gesprochen, Nachfragen ins Abstrakte oder Ungefähre verwischt. Zudem hat er vor seiner Feldforschung jegliche ,identifizierenden ' Bilder - mit ihm und seinem als solchen erkennbaren Partner oder mit dem damaligen Partner bei Pride-Veranstaltungen, usw. - in sozialen Medien gelöscht.

Während diese ,Vorsichtsmaßnahmen ' nötig erschienen, bedeuteten sie auch einen Eingriff in die Privatsphäre, eine Zensur nicht nur des beruflichen Daseins, sondern vor allem ein Übergriff auf die persönlichsten Beziehungen. Seither hat der Forscher einen zweiten Account auf Facebook für berufliche Kontakte aus Ruanda (da soziale Medien im ruandischen Kontext wichtig für die Kommunikation sind), der nicht mit anderen Kanälen verbunden ist. Die Notwendigkeit dieser Maßnahmen zeigte sich in einer Situation im Feld, in welcher er nach einem Interview informell mit dem Teilnehmenden Mittagessen war und das Gespräch auf Politikwechsel kam. Als Beispiel brachte der Forscher Angela Merkels erzwungenen Umschwung bezüglich der so genannten ,Ehe für alle` vor der politischen Sommerpause im Jahr 2017 aufs Tableau - also thematisch nicht auf seine eigene sexuelle Orientierung bezogen - und wurde darauf hingewiesen, ,,in diesem Land nie wieder über so etwas zu sprechen“. Aufgrund der ansonsten sehr liberal wirkenden Haltungen des Interviewten kann dieser Einwurf eher als Warnung und weniger als Drohung verstanden werden, aber die Stimmung im Gespräch war danach extrem aufgeladen und der Forscher empfand eine gewisse Bedrohung. Wie das Beispiel der Selbstzensur in sozialen Medien zeigt, ist die Gefahrenlage nicht nur durch das Verhalten im Feld, sondern auch von Handlungen vor und zwischen Aufenthalten determiniert.

Für den Umgang mit solchen komplexen Szenarien der möglichen Gefährdung kann es keine allgemeingültigen Regeln geben, wie das Arbeiten in Kriegsgebieten oder ähnliches stattfinden soll. Daher bedürfen sie umso mehr einer kritischen Reflexion und eines kontextsensiblen Plans, um den Sicherheitsgefahren zu begegnen. Im Folgenden eruieren wir, ob und inwiefern standardisierte Ethikkommissionen bei diesem Umgang mit Sicherheitsrisiken in solchen Forschungsprojekten hilfreich sein könnten. 


\section{Für und Wider von standardisierten Ethikkommissionen}

Wie in der Einleitung erwähnt, unterscheiden sich Entwicklungen zu standardisierten Ethikkommissionen und Reviews im angelsächsischen und deutschsprachigen Wissenschaftsraum. Im angelsächsischen Raum, in dem solche Kommissionen weit verbreitet sind, diskutieren insbesondere Sozialwissenschaftler*innen auch über Konflikt- und Fluchtbezug hinaus die Rolle dieser standardisierten Kommissionen und Reviews kritisch. Unter solchen standardisierten Kommissionen verstehen wir institutionalisierte Arbeitsgruppen mit Sanktionsmacht, die standardisierte Werkzeuge zur forschungsethischen Überprüfung von Projekten nutzen. Dieser Debatten bedienen wir uns hier. Einige Wissenschaftler*innen hinterfragen gar die generelle Notwendigkeit der Prüfungen, da sozialwissenschaftliche verglichen mit medizinischer Forschung selten Schäden verursache (Dingwall 2006, S. 52; Haggerty 2004, S. 399; Schrag 2011, S. 124) und die Nichteinhaltung von Regularien ohnehin keine Konsequenz habe (Haggerty 2004, S. 410; Murphy und Dingwall 2007, S. 2231).

Argumente gegen institutionalisierte Kontrollen von Forschungsethik in den Sozialwissenschaften reichen von unverhältnismäßigen Verfahren (Richardson und McMullan 2007, S. 1124) über Irrelevanz (Schrag 2011, S. 124) bis hin zur Einschränkung der Freiheit der Wissenschaft durch ein oktroyiertes Diktat. Am schärfsten formuliert es Dingwall, der kritisiert, dass staatliche Ethikkommissionen zur größten Bedrohung der Sozialwissenschaften und ihrer eigentlichen Rolle in demokratischen Gesellschaften würden, da staatlich gelenkte Mechanismen zur „Verschwendung öffentlicher Gelder, zu defizitären Informationen für Bürger*innen und langfristig zum wirtschaftlichen und politischen Niedergang" beitrügen (übers. von Verf., Dingwall 2007, S. 794). Dabei argumentiert er wie auch andere Forschende, dass Ethikkommissionen auf biomedizinischen Modellen fußten und nicht zu den häufig qualitativen und explorativen Sozialwissenschaften passten (Murphy und Dingwall 2007, S. 2230; Schrag 2011, S. 121; Wynn 2011, S. 103). Ähnlich bemängeln weitere, dass Kommissionen „scientific governance“ (Braun et al. 2010, S. 859) betrieben. Ethikprüfungen würden eine Normierung der Forschung (van den Hoonaard 2001, S. 21) und Zensur (Dingwall 2006, S. 54) verhängen und mehr Regularien würden Qualität und forschungsethische Standards nicht erhöhen, sondern ggf. verschlechtern (Hammersley 2009). Bezüglich unserer in diesem Artikel diskutierten Sicherheitsbelange gilt hier auch ganz besonders die Frage, inwieweit Sicherheitslagen und -probleme über verschiedene Fälle und Projekte hinweg überhaupt vergleichbar und ob standardisierte Risikoabwägungen hier hilfreich sind.

Prüfungen können zudem unangemessenen und unverhältnismäßigen psychischen Druck auf Forschende auslösen (Wynn 2011, S. 95). Die komplexen Prozeduren und Angst vor einer Nicht-Zustimmung können dazu führen, dass Forschende die kritischen Probleme verschweigen, um nicht die Genehmigung für ihre Projekte zu gefährden. Hier wäre beispielsweise die Sexualität des Forschenden zwar ein sicherheitsrelevantes Thema, aber eins, welches - aus Sorge vor einer zu vorsichtigen Reaktion einer Kommission - einfach verschwiegen bzw. vor dem Hintergrund der Nicht-Diskriminierung zur Seite geschoben werden könnte. Ähnliches könnte für die Forschung über sexuelle Gewalt an Geflüchteten angenommen werden, wenn diese nicht nur Frauen als Opfer betrachtet - lokale Bedingungen und Entwick- 
lungen lassen sich zwar vorab einschätzen, eine tatsächliche Bewertung ist jedoch erst vor Ort möglich. Nichtsdestotrotz eignen sich eben diese Themen besonders für einen engen Austausch und weitgehende Reflexionen mit Kolleg*innen - oder fordern diese förmlich. Die mögliche Geheimhaltung der eigenen Sexualität oder auch potenzieller Gefahren im Zuge der Gewaltforschung vor einer interventionsfähigen Ethikkommission wäre hier wahrscheinlich, da die komplexe Problematik außerhalb normbezogener Bewertungsschemata zu diskutieren wäre. Dies wird einer Kommission, die auf juristische Absicherung und standardisierte Lösungen fokussiert ist, ggf. nicht zugetraut. Allerdings wäre die Thematisierung dieser Sicherheitsbedenken eigentlich wichtig und sie bedürften einer fundierten Reflexion. Beiden Autor*innen ist es nur durch den Austausch mit Kolleg*innen gelungen, Lösungen zu finden, welche einerseits sowohl ihre Sicherheit als auch ihr Sicherheitsgefühl erhöht haben. Andererseits wurde ein Vorgehen identifiziert, dass der Sicherheit der Teilnehmenden in der Forschung über Gewalt zuträglich war. Ein strukturierter Rahmen hierfür - wenngleich ohne Sanktionierungsmacht - hätte das schneller und vermutlich noch besser aufgefangen.

Im Gegensatz dazu ist ein zentrales Argument für institutionalisierte Ethikprüfungen, dass forschungsethische Standards fundamentale Handlungskodizes bieten und die Schadensvermeidung anstreben (Brounéus 2011, S. 150; de Laine 2000, S. 144; Hedgecoe 2008, S. 880), wobei eine Prüfung eine Qualitätskontrolle anhand eben dieser Prinzipien darstellt (APSA 2012, S. 1; Schönhuth et al. 2001). Laut Witte und Heitkamp (2005, S. 2) können Ethikkommissionen als multidisziplinäre Beiräte unter anderem Beratungen leisten, Empfehlungen aussprechen und Informationen bereitstellen sowie die Einhaltung gewisser Normen kontrollieren, wobei der Austausch mit Kommissionsmitgliedern befruchtend für Forschung und Forschende sei (Hedgecoe 2008, S. 879). Insbesondere in Studien, in denen mit Personen in Konflikten, Postkonflikt- oder Flüchtlingssituationen geforscht wird, ist die Einhaltung von forschungsethischen Verhaltenskodizes zentral, wobei Reflexionsprozesse durch Ethikrichtlinien von und Diskussionen mit Ethikkommissionen als wertvoll für die Umsetzung der Feldforschung erachtet werden (Hoglund und Ober 2011, S. 195). Auch in unserem hier diskutierten Fall der Sicherheit ist eine solche konsequente Normenkontrolle wichtig. Die Individualität der Sicherheitsbedürfnisse und die starke Unterschiedlichkeit der verschiedenen Kontexte der Feldforschung erfordern indes einen flexibleren, für den jeweiligen Kontext geeigneten Katalog an Regeln, damit die fundamentalen Werte gewahrt, aber zielorientiert und kontextabhängig angepasst werden können. So wäre mit der Sexualität von Forschenden und Interviewpartner*innen in unterschiedlichen Kontexten gänzlich anders umzugehen, eng verbunden mit einer Reihe von Faktoren, die stark variieren können: Persönlichkeit und Hintergrund der Forschenden und Interviewpartner*innen, politischer, juristischer und gesellschaftlicher Rahmen, Vulnerabilität der Forschenden und Interviewpartner*innen, Thema der Forschung und Grad der politischen Brisanz, genauer geographischer Einsatz, und viele mehr.

Wir nehmen die Argumente für und gegen standardisierte Kommissionen und Reviews ernst und unsere Positionierung in der Debatte zeigt eine klare Tendenz für forschungsethische Reflexionen, die nicht notwendigerweise in höchst formalisierten Verfahren geprüft werden müssten. Dem Urteil, sozialwissenschaftliche 
Forschung würde selten Schädigungen verursachen, folgen wir indes nicht, da wie oben geschildert - tatsächliche Gefahren und Schädigungen zweifelsohne möglich sind und vor allem auch die Perspektive der Teilnehmenden wichtig ist. So entgegnen auch weitere Forschende der Annahme, sozialwissenschaftliche Studien verursachten keine Risiken, dass sie diese werteten und Folgen unterschätzten, und dass Ethikkommissionen relevant für jedwede Reflexion und Minimierung von Schädigungen seien (Hedgecoe 2008, S. 880; Nicholls et al. 2012, S. 72). Auch die Kritik, die Kommissionen folgten generell medizinischen Modellen, wird als Fehlinterpretation der Strukturen eingeschätzt, da diverse wissenschaftliche Disziplinen und Ansätze repräsentiert und berücksichtigt seien (Hedgecoe 2008, S. 874, 878).

In dieser breiten Diskussion über Ethikverfahren zielen die meisten Argumente gegen standardisierte Kommissionen und Prüfungen, nicht aber gegen Forschungsethik an sich. Konkrete Kritikpunkte betreffen institutionelle Rahmen, bürokratische Verfahren und formalisierte Regeln, die die Forschungsprojekte und Forschenden limitieren, Zensuren verhängen und nicht ausreichend auf inter- und multidisziplinäre Besonderheiten und Gegebenheiten eingehen. Da allerdings eine kritische Informationsweitergabe über fundamentale Handlungskodizes, Bewusstseinsschaffung und allem voran Austauschmöglichkeiten auch als positiv bewertet werden, bietet sich unseres Erachtens eine alternative Form des Gremiums an, welches sich insbesondere forschungsethischen Fragen und ihren Reflexionen widmen würde.

\section{Flexible interdisziplinäre Ethikgremien als mögliche Alternative}

Da wir Forschungsethik in der Feldforschung nicht nur als Grundlage für Verhaltenskodizes, sondern vor allem auch als normengeleitete Reflexionen der Zugänge und Methoden zur Datenerhebung verstehen, erachten wir die Bereitstellung von Informationen und die Förderung von Abwägungsprozessen als den besonderen Wert von fest institutionalisierten Ethikkommissionen, der auch ihre Notwendigkeit unterstreicht. Die obige Diskussion der Argumente für und gegen solche Kommissionen zeigt jedoch die bestehende Kritik an der Ausgestaltung der Kommissionen mit einer starken Kontrollfunktion, die mehr zu Zensur und Verbot als zu Reflexion anregt. Zudem haben Ethikkommissionen im angelsächsischen Raum starke Eingriffsmöglichkeiten in Projekte, die insbesondere explorativ ausgerichtete Forschungen einschränken können. Hiermit sind solche Kommissionen nicht besonders geeignet im Umgang mit sensiblen Problemen wie die Sicherheitsbelange, die wir in diesem Artikel exemplarisch diskutieren.

Um aus den Kritikpunkten zu lernen und die ethisch verantwortliche Durchführung empirischer Forschung zu befördern, stellen wir ein Modell einer flexibleren Herangehensweise vor: flexible interdisziplinäre Ethikgremien. Einhergehend mit ähnlich gelagerten Ansätzen (vgl. u. a. Dittmer und Lorenz 2018; van den Hoonaard und Hamilton 2016) sieht unser Ansatz eine institutionell gestützte Berücksichtigung von Forschungsethik in empirischer Forschung vor, welche Forschende unterstützt und Reflexion fördert, ohne Zensuren zu verhängen und wissenschaftliche Freiheit zu begrenzen. Während sich Dittmer und Lorenz (2018) etwa für eine ,reziproke Ethik der Vulnerabilität“ anstatt institutionalisierter Kommissionen für die Katastro- 
phenforschung aussprechen, stellen wir Diskussionsmöglichkeiten mit Blick auf die Gremien in den Mittelpunkt. So erhalten Forschende die Möglichkeit des Austausches über Ethikfragen, Werkzeuge und Handlungsrahmen, mit denen sie eigenverantwortlich und reflexiv mit Menschen ,im Feld' interagieren können. Aufgrund der unterschiedlichen Bedingungen und Herausforderungen der Projekte müssen die Forschenden die forschungsethischen Instrumente jeweils angepasst nutzen.

Unser Vorschlag flexibler, interdisziplinärer Ethikgremien ist nicht im engen Sinne als Kontrollinstanz mit weitreichenden Verbotskompetenzen gedacht, sondern soll in erster Linie zu einem kritisch-reflektierten Umgang mit der eigenen Forschungspraxis motivieren. Daher wird das Gremium primär in einer Beratungs- und Unterstützungsfunktion gesehen. Entsprechend kann ein solches Gremium durch seine Verfahren den Reflexionsprozess von Forschenden anleiten, indem bestimmte forschungsethische Kernbereiche abgefragt und mit sinnstiftenden Fragen reflektiert werden. Dies geht mit aktuellen Debatten einher, denn etwa auch der Rat für Sozialund WirtschaftsDaten (2017, S. 24) empfiehlt, ,[u]m der Komplexität forschungsethischer Anforderungen gerecht zu werden, [...] durch Angebote auf verschiedenen Ebenen eine Infrastruktur zur forschungsethischen Reflexion zu etablieren“.

Durch diese angeleitete Reflexion können kritische Themen der geplanten Forschung zum Vorschein kommen. Dies betrifft nicht nur Aspekte, wie die von uns exemplarisch vorgebrachten Sicherheitsfragen, sondern ggf. auch jene, die in der Gremiumsreflexion aufkommen und über die die Forschenden noch nicht nachgedacht hatten. Die Diskussion kann dann zur Erarbeitung von potenziellen Lösungsstrategien im Reflexionsprozess beitragen. Sollten diese nicht adäquat erscheinen, können sie im Dialog mit dem Gremium weiterentwickelt werden. Der Charakter des Gremiums ist also eher ein befruchtender Erfahrungsaustausch, in dem Gremienmitglieder ihre Ideen und Erfahrungen einbringen, als dass sie restriktiv und begrenzend agieren.

Da Forschungsethik für Feldforschung in der Konflikt- und Fluchtforschung bisher nicht immer im erforderlichen Maße berücksichtigt wird, aber aufgrund des Feldforschungs-Booms ein unweigerlicher Aufholbedarf besteht, sehen wir das Konzept der flexiblen Ethikgremien als wertvoll an. Durch Mitglieder aus diversen Disziplinen und mit internationaler Forschungserfahrung können in konstruktiver Diskussion vielfältige konkrete und ggf. praktische Fragen eruiert werden, die für die Arbeit in herausfordernden Kontexten in der Konflikt- und Fluchtforschung hilfreich sind. Ohne Machthierarchien aufzubauen, wird ein konstruktives Gespräch auf Augenhöhe ermöglicht, aus dem problemlösungsorientierte Konsequenzen folgen können. So lassen sich beispielsweise verschiedene Strategien diskutieren, wie die persönliche Sicherheit der Forschenden gewährleistet sein kann, ohne dass dies einen formalen Charakter annimmt, bei welchem eine Abweichung im Moment der Feldforschung dienstliche oder sogar juristische Folgen haben könnte. Entsprechend erachten wir einerseits den intensiven Austausch über Forschungsethik und andererseits flexible Ethikverfahren zur Prüfung von Konzepten und für Austauschmöglichkeiten als erforderlich. Vor dem Hintergrund der unterschiedlichen Disziplinen, der qualitativen und quantitativen Methodenvielfalt und der Notwendigkeit, diese informiert in Prüfungen zu berücksichtigen, sind flexible forschungsethische Infrastrukturen zielführend, in denen Ethikfragen mit versierten Ansprechpartner*innen diskutiert und 
richtungsweisende Rahmen bereitgestellt werden können (Richardson und McMullan 2007, S. 1128; von Unger et al. 2014, S. 31).

Ein Nachteil flexibler Ethikgremien besteht ggf. in eben jenen fehlenden Sanktionsmöglichkeiten bei Nicht-Einhaltung von Empfehlungen. Allerdings kritisieren Forschende auch an institutionellen Kommissionen, dass Prüfungen nur vor, aber nicht während des eigentlichen Forschungsprozesses stattfänden und ein Nicht-Einhalten ohnehin folgenlos sei. Die Flexibilität der Gremien soll vielmehr zur Teilnahme und zum Austausch anregen; vor allem können besonders kritische Themen diskutiert werden, anstatt sie auszuklammern, um ein Verbot zu vermeiden. Zweifelsohne erfordert dies ein hohes Maß an Selbstverantwortung in der Implementierung. Dies ist jedoch bei Feldforschung generell notwendig und der reflexive Prozess flexibler Ethikgremien könnte die Selbstreflexion und -verantwortung unterstützen.

Diese Reflexivität ist in der Struktur begründet, die basierend auf Guillemin und Gillam (2004) in die ,prozedurale Ethik ‘ und die ,Ethik in der Praxis ‘ unterteilt wird, wobei rahmende Ethikrichtlinien die Grundlage darstellen. Die ,prozedurale Ethik bezieht sich auf eine Begutachtung der Vorhaben anhand eines Konzepts durch eine Kommission vor der Feldforschung (Guillemin und Gillam 2004, S. 263). Die ,Ethik in der Praxis' berücksichtigt Besonderheiten in den Forschungsfeldern und von Forschungsprozessen im Feld, sie bezieht sich also auf die ,alltäglichen ethischen Herausforderungen, die bei der Durchführung der Forschung aufkommen“ (Guillemin und Gillam 2004, S. 263). Für die ,Ethik in der Praxis“ sind ethische Kompetenzen der Forschenden zentral, womit Guillemin und Gillam (2004, S. 269) auf die Bereitschaft von Forschenden eingehen, die ethischen Dimensionen der Forschungspraxis anzuerkennen sowie ihre Fähigkeiten, die Dimensionen zu verstehen, zu reflektieren und entsprechend darauf zu reagieren. Dafür sind die Selbstreflexion der eigenen Rolle im Feld sowie der Respekt gegenüber Teilnehmenden wesentlich. Es werden kontextabhängige Bedingungen und ethisch unvorhersehbare Fälle berücksichtigt, die ,ethisch wichtige Momente“ darstellen (Guillemin und Gillam 2004; vgl. auch Block et al. 2012, S. 70), aber keine oder nur bedingte Beachtung in prozeduralen Ethikprüfungen finden können. Im Erfahrungsaustausch können somit ungewisse Situationen durchdacht und mögliche Reaktionen auf bestimmte Eventualitäten erarbeitet und vorbereitet werden. Auch wenn hier sicherlich nicht alle ethisch relevanten Situationen bedacht werden können, wird sich ein Trainingseffekt einstellen, indem das strukturierte Nachdenken über das Problem und ein ethisch sensibler und lösungsorientierter Umgang damit geübt wird.

\section{Abschließende Gedanken}

Das Ziel des Beitrags war es, die zentrale Stellung von Forschungsethik für die Feldforschung insbesondere mit Blick auf institutionalisierte Ethikkommissionen und -prüfungen zu reflektieren. Auf der Grundlage unserer Beispiele zur Gefährdung der Sicherheit von Teilnehmenden und Forschenden sowie der Debatte über das Für und Wider von standardisierten Kommissionen und Prüfverfahren haben wir einen Vorschlag für flexible, interdisziplinäre Ethikgremien entwickelt, die vor allem als Anlaufstellen für weiterführenden Austausch zur Forschungspraxis zur Verfügung 
stehen könnten. Obgleich - oder sogar weil - die Gremien keine Kontrollfunktionen bei der Durchführung der Projekte haben würden, erhalten Forschende Richtlinien, Hinweise und Reflexionsmöglichkeiten, die wertvoll für den Forschungsprozess sind. Eben diese Bedeutung des Austauschs haben wir anhand eigener Erfahrungen eruiert. Unsere Beispiele zum Umgang mit Gefährdung von Forschenden und Teilnehmenden aufgrund sexueller Orientierung sowie genderbasierter Gewalt zeigen, wie wichtig die Reflexionsräume sind, die durch flexible Ethikgremien, nicht aber durch institutionalisierte Kontrollinstanzen, bereitgestellt werden können.

Weiterführende Fragen zum Nutzen dieser Gremien betreffen nicht nur ihre tatsächliche Form, sondern auch ihre internationale Anerkennung. Einerseits ist denkbar, dass diese Gremien in den jeweiligen universitären Institutionen oder überuniversitär an der Arbeitsgemeinschaft für Friedens- und Konfliktforschung e. V. sowie dem Netzwerk Fluchtforschung e. V. angebunden werden. Letzteres bietet die Möglichkeit Austauschmöglichkeiten mit Kolleg*innen zu nutzen, die zu ähnlichen Themen forschen und entsprechend mit ähnlichen Herausforderungen vertraut sind. Die Mitglieder der Gremien würden unterschiedliche Disziplinen, Forschungsschwerpunkte, Methodenkenntnisse und regionale Interessensfelder vertreten, um der Multi- und Interdisziplinarität sowie der Vielfalt der Methoden und Projektthemen in beiden Bereichen gerecht zu werden, und als Ansprechpartner*innen agieren zu können. ${ }^{9}$ Andererseits sind jedoch Ethikprüfungen international erforderlich. Die positiven Prüfergebnisse werden zunehmend von Journals in der englischsprachigen Publikation von Artikeln oder bei Anträgen für Drittmittel benötigt. Dies stellt Sozialwissenschaftler*innen in Deutschland teilweise vor Herausforderungen. Ob und wie die vorgeschlagenen flexiblen Ethikgremien auch Prüfverfahren vornehmen könnten oder inwiefern die pure Reflexion als Indiz für umfassende forschungsethische Abwägung verstanden werden könnte, sind Fragen, die weiterführender Diskussionen bedürfen und die wir an dieser Stelle nicht abschließend beantworten können - dies nicht zuletzt auch, weil die Gremien zunächst pilotiert werden müssten.

In unserem Beitrag haben wir den aktuellen Feldforschungs-Boom in der Friedens- und Konfliktforschung sowie der Flucht- und Flüchtlingsforschung als relativ gegeben hingenommen und Vorschläge unterbreitet, wie in diesen Feldern ethische Prinzipien gewährleistet werden können. Unser Plädoyer für interdisziplinäre, flexible Ethikgremien sollte verstanden werden als Kompromiss zwischen einer nötigen Freiheit der Wissenschaft, d.h. einem Grundvertrauen in den kompetenten Umgang mit dem eigenen Forschungsbereich und Kenntnis des eigenen Falls auf der einen Seite sowie den Möglichkeiten einer angeleiteten Reflexion und der Unterstützung und Beratung durch peers auf der anderen Seite. So wird es ermöglicht, dass nicht jede*r Forschende stets das Rad neu erfinden muss, sondern auf den Rat von peers zurückgreifen und auf ethisch reflektierte Praxen anderer aufbauen kann. Hierdurch würde die deutschsprachige Forschungslandschaft von einem in ethischer Hinsicht nahezu ambivalenten Raum zu einem Ort tiefer Reflexion gelangen - dies bedeutet nicht, dass diese Reflexion bei den meisten Wissenschaftler*innen nicht schon

\footnotetext{
${ }_{9}$ Das bedeutet auch, dass limitierende Entscheidungen und Diktate vermieden werden, denn die Personen sind nicht fachfremd, sondern können Kontexte und Herangehensweisen generell abschätzen.
} 
stattfindet, aber so wird der Weg dafür geebnet, dass dies in einem konzertierten Verfahren und in einer strukturierten Weise abläuft.

Funding Open Access funding provided by Projekt DEAL.

Open Access Dieser Artikel wird unter der Creative Commons Namensnennung 4.0 International Lizenz veröffentlicht, welche die Nutzung, Vervielfältigung, Bearbeitung, Verbreitung und Wiedergabe in jeglichem Medium und Format erlaubt, sofern Sie den/die ursprünglichen Autor(en) und die Quelle ordnungsgemäß nennen, einen Link zur Creative Commons Lizenz beifügen und angeben, ob Änderungen vorgenommen wurden.

Die in diesem Artikel enthaltenen Bilder und sonstiges Drittmaterial unterliegen ebenfalls der genannten Creative Commons Lizenz, sofern sich aus der Abbildungslegende nichts anderes ergibt. Sofern das betreffende Material nicht unter der genannten Creative Commons Lizenz steht und die betreffende Handlung nicht nach gesetzlichen Vorschriften erlaubt ist, ist für die oben aufgeführten Weiterverwendungen des Materials die Einwilligung des jeweiligen Rechteinhabers einzuholen.

Weitere Details zur Lizenz entnehmen Sie bitte der Lizenzinformation auf http://creativecommons.org/ licenses/by/4.0/deed.de.

\section{Literatur}

American Anthropological Association (AAA). 2012. Principles of professional responsibility. http:// ethics.aaanet.org/category/statement/. Zugegriffen: 8. Apr. 2020.

American Political Science Association (APSA). 2012. A guide to professional ethics in political science. http://www.apsanet.org/RESOURCES/For-Faculty/Ethics. Zugegriffen: 8. Apr. 2020.

Banks, Sarah, et al. 2013. Everyday ethics in community-based participatory research. Contemporary Social Science 8(3):263-277.

Block, Karen, et al. 2012. Addressing ethical and methodological challenges in research with refugeebackground young people: reflections from the field. Journal of Refugee Studies 26(1):69-87.

Braun, Kathrin, et al. 2010. Ethical reflection must always be measured. Science, Technology \& Human Values 35(6):839-864.

British Sociological Association (BSA). 2017. Statement of ethical practice for the British sociological association. https://www.britsoc.co.uk/media/24310/bsa_statement_of_ethical_practice.pdf. Zugegriffen: 8. Apr. 2020.

Brounéus, Karen. 2011. In-depth interviewing: the process, skill and ethics of interviews in peace research. In Understanding peace research: methods and challenges, Hrsg. Kristine Hoglund, Magnus Ober, 130-146. London: Routledge.

Browne, Brendan, und Luke Moffett. 2014. Finding your feet in the field: critical reflections of early career researchers on field research in transitional societies. Journal of Human Rights Practice 6(2):223-237.

Clark-Kazak, Christina R., et al. 2017. Ethical considerations: research with people in situations of forced migration. Refuge 33(2):11-17.

Darling, Jonathan. 2014. Emotions, encounters and expectations: the uncertain ethics of 'the field'. Journal of Human Rights Practice 6(2):201-212.

Deutsche Gesellschaft für Erziehungswissenschaften (DGfE). 2016. Ethik-Kodex der Deutschen Gesellschaft für Erziehungswissenschaft. https://www.dgfe.de/service/ethik-rat-ethikkodex.html. Zugegriffen: 8. Apr. 2020.

Deutsche Gesellschaft für Sozial- und Kulturanthropologie (DGSKA). 2019. Ethik Leitlinie. https://www. dgska.de/dgska/ethik/. Zugegriffen: 8. Apr. 2020.

Deutsche Gesellschaft für Soziologie (DGS). 2017. Satzung der Ethik-Kommission der Deutschen Gesellschaft für Soziologie (DGS) und des Berufsverbandes Deutscher Soziologinnen und Soziologen (BDS). https://soziologie.de/dgs/ethik/ethik-kodex. Zugegriffen: 8. Apr. 2020.

Dingwall, Robert. 2006. Confronting the anti-democrats: the unethical nature of ethical regulation in social science. Medical Sociology Online 1(1):51-58. 
Dingwall, Robert. 2007. "Turn off the oxygen ..." comment on the presidential address. Law \& Society Review 41(4):787-795.

Dittmer, Cordula, und Daniel F. Lorenz. 2018. Forschen im Kontext von Vulnerabilität und extremem Leid - Ethische Fragen der sozialwissenschaftlichen Katastrophenforschung. http://www.qualitativeresearch.net/index.php/fqs/article/view/3116/4279. Zugegriffen: 24. Juni 2020.

Doná, Giorgia. 2007. The microphysics of participation in refugee research. Journal of Refugee Studies 20(2):210-229.

Ellis, B. Heidi, et al. 2007. Ethical research in refugee communities and the use of community participatory methods. Transcultural Psychiatry 44(3):459-481.

Fluehr-Lobban, Carolyn. 2014. Ethics. In Handbook of methods in cultural anthropology, 2. Aufl., Hrsg. H. Russell Bernard, Clarence C. Gravlee, 131-150. London: Rowman \& Littlefield.

Gerver, Mollie. 2013. Exceptions to blanket anonymity for the publication of interviews with refugees: African refugees in Israel as a case study. Research Ethics 9(3):121-139.

Glammeier, Sandra. 2016. Gruppendiskussionen und dokumentarische Methode in der Forschung zu Gewalt. In Forschungsmanual Gewalt: Grundlagen der empirischen Erhebung von Gewalt in Paarbeziehungen und sexualisierter Gewalt, Hrsg. Cornelia Helfferich, Barbara Kavemann, und Heinz Kindler, 143-159. Wiesbaden: Springer.

Goodhand, Jonathan. 2000. Research in conflict zones. Ethics and accountability. Forced Migration Review 8:12-15.

Guillemin, Marilys, und Lynn Gillam. 2004. Ethics, reflexivity, and "ethically important moments" in research. Qualitative Inquiry 10(2):261-280.

Hagemann-White, Carol. 2016. Grundbegriffe und Fragen der Ethik bei der Forschung über Gewalt im Geschlechterverhältnis. In Forschungsmanual Gewalt: Grundlagen der empirischen Erhebung von Gewalt in Paarbeziehungen und sexualisierter Gewalt, Hrsg. Cornelia Helfferich, Barbara Kavemann, und Heinz Kindler, 13-31. Wiesbaden: Springer.

Haggerty, Kevin D. 2004. Ethics creep: governing social science research in the name of ethics. Qualitative Sociology 27(4):391-414.

Hammersley, Martyn. 2009. Against the ethicists: on the evils of ethical regulation. International Journal of Social Research Methodology 12(3):211-225.

Hedgecoe, Adam. 2008. Research ethics review and the sociological research relationship. Sociology 42(5):873-886.

Helfferich, Cornelia. 2016. Qualitative Einzelinterviews zu Gewalt: Die Gestaltung der Erhebungssituation und Auswertungsmöglichkeiten. In Forschungsmanual Gewalt: Grundlagen der empirischen Erhebung von Gewalt in Paarbeziehungen und sexualisierter Gewalt, Hrsg. Cornelia Helfferich, Barbara Kavemann, und Heinz Kindler, 121-142. Wiesbaden: Springer.

Hoglund, Kristine, und Magnus Ober. 2011. Improving information gathering and evaluation. In Understanding peace research: methods and challenges, Hrsg. Kristine Hoglund, Magnus Ober, 185-198. London: Routledge.

van den Hoonaard, Will C. 2001. Is research-ethics review a moral panic? Canadian Review of Sociology/ Revue canadienne de sociologie 38(1):19-36.

van den Hoonaard, Will C., und Ann Hamilton. 2016. The ethics rupture: exploring alternatives to formal research-ethics review. Toronto: University of Toronto Press.

Hugman, Richard, Linda Bartolomei, und Eileen Pittaway. 2011a. Human agency and the meaning of informed consent: reflections on research with refugees. Journal of Refugee Studies 24(4):655-671.

Hugman, Richard, Eileen Pittaway, und Linda Bartolomei. 2011b. When 'do no harm' is not enough: the ethics of research with refugees and other vulnerable groups. British Journal of Social Work 41(7):1271-1287.

International Association for the Study of Forced Migration (IASFM). 2018. Code of ethics: critical reflections on research ethics in situations of forced migration

Jacobsen, Karen, und Loren B. Landau. 2003. The dual imperative in refugee research: some methodological and ethical considerations in social science research on forced migration. Disasters 27(3):185-206.

Krause, Ulrike. 2017. Researching forced migration. Critical reflections on research ethics during fieldwork. RSC working paper series, Bd. 123. Oxford: RSC.

de Laine, Marlene. 2000. Fieldwork, participation and practice: ethics and dilemmas in qualitative research. London: SAGE.

Van Liempt, Ilse, und Veronika Bilger. 2012. Ethical challenges in research with vulnerable migrants. In Handbook of research methods in migration, Hrsg. Carlos Vargas-Silva, 451-566. Cheltenham: Edward Elgar. 
Mackenzie, Catriona, Christopher McDowell, und Eileen Pittaway. 2007. Beyond 'do no Harm': the challenge of constructing ethical relationships in refugee research. Journal of Refugee Studies 20(2):299-319.

Makofane, Keletso, et al. 2014. Homophobic legislation and its impact on human security. African Security Review 23(2):186-195.

Malkki, Liisa H. 1995. Purity and exile: violence, memory, and national cosmology among Hutu refugees in Tanzania. Chicago, London: University of Chicago Press.

Marlowe, Jay M., et al. 2015. Conducting post-disaster research with refugee background peer researchers and their communities. Qualitative Social Work 14(3):383-398.

Menzel, Anne. 2014. Zwischen Herrschaftswissen und Irrelevanz? Feldforschung und das Ringen mit der Policy-Relevanz. Zeitschrift für Friedens- und Konfliktforschung 3(2):264-283.

Murphy, Elizabeth, und Robert Dingwall. 2007. Informed consent, anticipatory regulation and ethnographic practice. Social Science \& Medicine 65(11):2223-2234.

Nicholls, Stuart G., Jamie Brehaut, und Raphae Saginur. 2012. Social science and ethics review: A question of practice not principle. Research Ethics 8(2):71-78.

Pittaway, Eileen, und Linda Bartolomei. 2013. Doing ethical research: 'whose problem is it anyway? In Values and vulnerabilities. The ethics of research with refugees and asylum seekers, Hrsg. Karen Block, Elisha Riggs, und Nick Haslam, 151-170. Toowong: Australian Academic.

Pittaway, Eileen, Linda Bartolomei, und Richard Hugman. 2010. 'Stop stealing our stories': the ethics of research with vulnerable groups. Journal of Human Rights Practice 2(2):229-251.

Rat für Sozial- und WirtschaftsDaten. 2017. Forschungsethische Grundsätze und Prüfverfahren in den Sozial- und Wirtschaftswissenschaften. 9 Output 5. Berufungsperiode. https://www.ratswd.de/dl/ RatSWD_Output9_Forschungsethik.pdf. Zugegriffen: 24. Juni 2020.

Refugee Studies Centre. 2007. Ethical guidelines for good research practice. Refugee Survey Quarterly 26(3):162-172.

Richardson, Sue, und Miriam McMullan. 2007. Research ethics in the UK: what can sociology learn from health? Sociology 41(6):1115-1132.

Schneider, Luisa T. 2020. Sexual violence during research: How the unpredictability of fieldwork and the right to risk collide with academic bureaucracy and expectations. Critique of Anthropology https:// doi.org/10.1177/0308275X20917272.

Schönhuth, Michael, Frank Bliss, und Sandra Wentzel. 2001. Ethical guidelines of the workgroup development anthropology (AGEE) e. V. Explanations and advice. Trierer Reihe: Materialien zur Ethnologie.

Schrag, Zachary M. 2011. The case against ethics review in the social sciences. Research Ethics 7(4):120-131.

Schulz, Philipp. 2018. Displacement from gendered personhood: sexual violence and masculinities in northern Uganda. International Affairs 94(5):1101-1119.

Siegel, Dina, und Roos de Wildt (Hrsg.). 2016. Ethical concerns in research on human trafficking. Studies of organized crime, Bd. 13. Berlin, Heidelberg, New York: Springer.

Sivakumaran, Sandesh. 2005. Male/Male rape and the "taint" of homosexuality. Human Rights Quarterly 27(4):1274-1306.

Thomson, Susan, An Ansoms, und Jude Murison (Hrsg.). 2013. Emotional and ethical challenges for field research in africa: the story behind the findings. Basingstoke: Palgrave Macmillan.

von Unger, Hella, und Dagmar Simon. 2016. Ethikkommissionen in den Sozialwissenschaften - Historische Entwicklungen und Internationale Kontroversen. RatSWD working paper, Bd. 253

von Unger, Hella, Petra Narimani, und Rosaline M'Bayo. 2014. Einleitung. In Forschungsethik in der qualitativen Forschung, Hrsg. Hella von Unger, Petra Narimani, und Rosaline M'Bayo, 1-14. Wiesbaden: Springer.

Vervliet, Marianne, et al. 2015. Multilayered ethics in research involving unaccompanied refugee minors. Journal of Refugee Studies 28(4):468-485.

Witte, Erich H., und Imke Heitkamp. 2005. Empirical research on ethics: the influence of social roles on decisions and on their ethical justification. Bd. 61

Wood, Elisabeth Jean. 2006. The ethical challenges of field research in conflict zones. Qualitative Socio$\log y$ 29(3):373-386.

Wynn, Lisa L. 2011. Ethnographers' experiences of institutional ethics oversight: results from a quantitative and qualitative survey. Journal of Policy History 23(1):94-114. 